\section{Rede FIBRA-RJ: fragilidade e risco de hospitalização em idosos da cidade do Rio de Janeiro, Brasil}

\author{
FIBRA-RJ Network: frailty and risk of \\ hospitalization in the elderly in \\ Rio de Janeiro, Brazil \\ Red FIBRA-RJ: la fragilidad y el riesgo de \\ hospitalización para las personas mayores \\ en la ciudad de Río de Janeiro, Brasil
}

Mariangela Perez 1

Roberto Alves Lourenço 1

\author{
1 Universidade do Estado do \\ Rio de Janeiro, Rio de Janeiro, \\ Brasil. \\ Correspondência \\ M. Perez \\ Universidade do Estado do \\ Rio de Janeiro \\ Av. Marechal Rondon 381 \\ Rio de Janeiro, RJ 20950-003, \\ Brasil. \\ mariangela.perez@hotmail. \\ com
}

\section{Abstract}

The objective of this study was to determine the risk profile and factors associated with frailty in elderly community residents. The population consisted of individuals 65 years or older living in the northern districts of the city of Rio de Janeiro, Brazil, and who held private health insurance policies. The cross-sectional study was done at baseline in a cohort with a sample $(N=764)$ stratified by gender and age. Risk stratification used probability of repeated admissions (PRA) as the screening instrument. Following bivariate analyses, logistic regression analyses were performed to study associations between probability of repeated admissions and socio-demographic, health-status, functional, and cognitive variables. Of the total sample, 6.7\% were classified as high risk. Cancer, falls, chronic obstructive pulmonary disease, use of medication, receiving a visit from a health professional, being bedridden at home, living alone, and level of activities of daily living were statistically associated with risk of hospitalization. The instrument appeared to be useful for stratifying risk in the elderly.

Hospitalization; Aging; Health of the Elderly; Health Services

\section{Resumo}

O objetivo do estudo foi determinar o perfil de risco e fatores associados à fragilidade em idosos da comunidade. A população-fonte constituiuse de indivíduos com 65 anos ou mais, residentes nos bairros da zona norte da cidade do Rio de Janeiro, Brasil, e clientes de uma operadora de saúde. O estudo foi transversal, na linha de base de uma coorte, com amostra estratificada por sexo e idade, composta por 764 indivíduos. Para a estratificação de risco, utilizou-se o instrumento de rastreio probabilidade de internações repetidas (PIR). A análise de regressão logística foi realizada para estudar a associação entre a PIR $e$ um conjunto de variáveis sociodemográficas, de estado de saúde, funcionais e cognitivas, após a análise bivariada. Encontraram-se 6,7\% de idosos com alto risco de internação. Associaram-se ao risco de internação câncer, quedas, doença pulmonar obstrutiva crônica e medicamentos usados, bem como as seguintes condições: receber visita de profissional da saúde, ter estado acamado no domicílio, morar só e praticar as atividades de vida diária. O instrumento parece ser útil na estratificação de risco dos idosos.

Hospitalização; Envelhecimento; Saúde do Idoso; Serviços de Saúde 


\section{Introdução}

No Brasil, o Sistema Único de Saúde (SUS) é responsável por $80 \%$ da assistência de saúde, porém, segundo a Pesquisa Nacional por Amostra de Domicílios de 2008 (PNAD/2008), a cobertura pelo sistema suplementar vem aumentando, especialmente nas regiões Sul e Sudeste 1. Segundo dados da Agência Nacional de Saúde Suplementar (ANS), em junho de 2012, a taxa de cobertura no Brasil, para todas as faixas etárias, era de $25,1 \%$, equivalendo a aproximadamente 48 milhões de beneficiários, $11 \%$ dos quais tinham sessenta anos ou mais à época. Na Região Sudeste, a taxa de cobertura é de $38,4 \%$, sendo, no Estado do Rio de Janeiro, de $38,6 \%$ (ANS. Dados e indicadores do setor. http://www.ans.gov.br/materiais-para-pesqui sas/perfil-do-setor/dados-e-indicadores-do-se tor, acessado em 26/Out/2012). Portanto, o conhecimento das características de apresentação de morbidades dessa parcela da população é de grande relevância para adequação dos modelos de cuidado oferecido por esse setor.

O sistema de saúde no Brasil, tanto público, quanto privado, sofre a pressão da grande demanda por serviços, decorrente do envelhecimento populacional acelerado das últimas décadas. Por essa razão, para melhor planejamento do sistema, torna-se necessário desenvolver mecanismos de identificação do subgrupo de idosos que necessita de forma mais premente e intensa de serviços de saúde especializados, caracterizado na literatura da área como portadores da síndrome de fragilidade 2 .

Sabe-se que o idoso frágil faz parte do grupo com maior demanda de cuidados, consequente ao estado de maior vulnerabilidade, à baixa reserva homeostática e à reduzida capacidade de responder aos diferentes tipos de estresse. Esse quadro resulta em risco aumentado de eventos adversos, como, por exemplo, maior número de doenças agudas com lenta recuperação, quedas, dependência funcional, hospitalização, institucionalização e maior mortalidade 3 .

Não obstante a relação dessa síndrome com desfechos adversos de saúde estar claramente determinada, o conceito de fragilidade está ainda em evolução e, há pelo menos trinta anos, propõem-se variados modelos teóricos, definições associadas e critérios operacionais que melhor se apliquem à síndrome, sem que, no entanto, um consenso seja atingido ${ }^{4}$. De forma geral, foram utilizadas composições distintas de indicadores que avaliam mobilidade, força muscular, dependência para realizar as atividades de vida diária, cognição, morbidades, uso de serviços de saúde marcadores biológicos de inflamação e estado nutricional ${ }^{4}$. Além desses, as hospitalizações repetidas têm sido, frequentemente, consideradas indicadores de fragilidade 5, e vários modelos propostos utilizam a internação hospitalar como desfecho na construção de instrumentos de rastreio de idosos portadores de risco aumentado de eventos adversos de saúde 4 .

$\mathrm{O}$ probability of repeated admission (PRA), proposto por Boult et al. 5, é um dos instrumentos mais utilizados para identificação de idosos frágeis ou com risco de fragilização, tanto nos Estados Unidos, como em outros países. Ele é simples e de rápida aplicação, e a sua validade foi determinada em populações com diferentes características 6,7,8,9,10,11,12. Contudo, no Brasil, ainda é necessário que estudos de validação sejam desenvolvidos antes de considerá-lo adequado para rastreamento de idosos frágeis em nossas instituições.

O conhecimento do perfil de fragilização de idosos residentes na comunidade, por meio da aplicação do PRA, mapeando os níveis de risco, pode contribuir não somente para a determinação da prevalência, mas também para o entendimento dos fatores relacionados às piores condições de saúde, permitindo planejar políticas mais adequadas às diferentes demandas da população, seja no sistema público, seja no sistema suplementar de saúde 13. Em um cenário de escassez de recursos humanos especializados na área de envelhecimento, a utilização de instrumentos que identifiquem, no nível de atenção primária de saúde, aqueles idosos que necessitam de abordagem específica e pautada na reabilitação pode ter grande aplicabilidade, vindo a ser útil para otimizar recursos humanos e financeiros 13 .

Este estudo teve como objetivo determinar o perfil de risco e os fatores associados à fragilidade em idosos residentes na zona norte da cidade do Rio de Janeiro, os quais fazem parte da carteira de clientes de uma operadora de saúde.

\section{Métodos}

Realizou-se um estudo transversal, descritivo, na linha de base da população que compõe o estudo Fragilidade em Idosos Brasileiros.

\section{População e amostra de estudo}

A população do estudo foi formada por indivíduos com idade igual ou superior a 65 anos, residentes em bairros da zona norte da cidade do Rio de Janeiro, que faziam parte do cadastro de clientes de uma fundação de autogestão de assistência de saúde e previdência, para servi- 
dores públicos federais e seus dependentes, com cobertura em diversos municípios brasileiros. A delimitação geográfica foi definida pela coordenação do projeto FIBRA-RJ, por conveniência logística. Essa população somava um total de 9.769 indivíduos, tomando como referência o mês de julho do ano de 2008.

A amostra foi selecionada pelo processo de estratificação segundo o cruzamento entre as variáveis faixa etária e sexo, formando dez estratos naturais, constituídos por indivíduos com idades de 65 até cem anos, divididos por faixas de dez anos. Cada estrato final foi obtido por meio de amostragem aleatória inversa, mantendo-se as proporções dos estratos da população-fonte, exceto para os indivíduos de 95 anos ou mais, os quais foram todos entrevistados. $\mathrm{O}$ tamanho da amostra foi calculado de forma que o coeficiente de variação em cada estrato natural fosse de $15 \%$ para estimativas de proporção em torno de 0,07, com nível de 95\% de confiança. O tamanho final da amostra foi obtido pela soma do número de indivíduos em cada estrato, totalizando 847 idosos.

Excluíram-se do estudo os idosos que necessitavam de um informante substituto por apresentarem alguma das seguintes condições: déficit cognitivo - definido por um resultado menor que 12 no Mini-Exame do Estado Mental (MEEM) 14; déficits sensoriais que comprometessem a comunicação e a leitura; doenças terminais de qualquer natureza. Entre outras limitações, o respondente substituto não responderia a autoavaliação de saúde, item que compõe o instrumento utilizado para estratificar o risco de fragilidade.

O recrutamento ocorreu, por meio telefônico, no período de 5 de janeiro de 2009 a 13 de janeiro de 2010.

\section{Aspectos éticos}

O estudo foi aprovado pelo Comitê de Ética em Pesquisa do Hospital Universitário Pedro Ernesto, da Universidade do Estado do Rio de Janeiro (1850-CEP/HUPE). Todos os participantes assinaram o Termo de Consentimento Livre e Esclarecido.

\section{A coleta de dados e o instrumento de pesquisa}

A coleta de dados foi realizada no domicílio em entrevista única, face a face, com duração de noventa minutos, aproximadamente, mediante o auxílio de um questionário com perguntas estruturadas e medidas de desempenho físico, funcional e mental.
O questionário foi composto de dados sociodemográficos, como situação conjugal e de moradia, escolaridade, cor da pele/raça, renda pessoal, idade, sexo e disponibilidade de um cuidador em caso de necessidade, e dados sobre o estado de saúde, por intermédio da autoavaliação de saúde e do autorrelato de doenças crônicas, como hipertensão arterial sistêmica (HAS), coronariopatia, diabetes mellitus, câncer, artropatias, doença pulmonar obstrutiva crônica, osteoporose, acidente vascular cerebral (AVC), déficits auditivo ou visual, além de quedas no último ano e incontinências urinária e fecal. $\mathrm{O}$ indivíduo foi questionado sobre o uso de serviços de saúde no último ano, caracterizado pelo número de internações e o tempo de permanência, número de consultas médicas, necessidade de visita domiciliar de profissionais de saúde, necessidade de permanecer acamado por motivo de doença e número de medicamentos utilizados regularmente nos três meses anteriores à entrevista. $\mathrm{O}$ estado mental foi avaliado pelo MEEM 14 - ponto de corte de 18/19 para analfabetos e 24/25 para os demais - e pela Escala de Depressão Geriátrica (EDG) 15 - ponto de corte $5 / 6$. A capacidade funcional foi avaliada pelas escalas de atividades instrumentais de vida diária (AIVD) de Lawton e de atividades básicas de vida diária (AVD) de Katz. Como essas escalas não têm pontos de corte definidos, considerouse, nas análises, o sentido de cada uma: a primeira pontua a independência, variando de 7 a 21; a segunda pontua a dependência, variando de zero a seis 16,17 . Foram realizadas medidas antropométricas, como peso e altura, e testes de desempenho, como velocidade da marcha média de três avaliações do tempo gasto para caminhar 4,6 metros em linha reta - e força de preensão manual - média de três medidas aferidas por dinamômetro, do tipo Jamar (SAEHAN Corporation, Yangdeok-Dong, Coreia do Sul), no membro superior dominante.

A probabilidade de internação repetida de cada participante foi calculada utilizando-se o PRA, composto por oito itens incluídos no questionário: (1) autoavaliação da saúde - com as seguintes opções de resposta: "muito boa, boa, regular, ruim ou muito ruim”; (2) internação no último ano; (3) número de consultas médicas no último ano; (4) presença de diabetes mellitus; (5) presença de doença coronariana; (6) sexo; (7) disponibilidade de um cuidador em caso de necessidade; (8) idade ${ }^{3}$. A equação logística e os coeficientes de regressão correspondentes a cada um dos oito itens foram descritos por Pacala et al. 9. No presente trabalho, o nome do instrumento, em língua portuguesa, passou a ser probabilidade de internações repetidas (PIR). 
Análise dos dados

\section{- Estratificação da amostra segundo o risco de internações}

A amostra foi classificada em dois estratos de risco segundo o valor da PIR, que varia de 0 a 1 . Consideraram-se como de alto risco os idosos com PIR $\geq 0,5$ 3. Dos oito itens que compõem o questionário, apenas a autoavaliação de saúde apresenta opções de resposta diferentes daquelas utilizadas no estudo original (excelente, muito boa, boa, regular, ruim), já que o presente trabalho adotou os padrões das pesquisas censitárias brasileiras. Os coeficientes de regressão originais foram mantidos e utilizados, de forma que "muito boa" substituiu "excelente"; "boa", "regular" e "ruim" não foram modificados e a "muito ruim" atribuiu-se o mesmo coeficiente de regressão que "ruim".

\section{- Análise estatística}

Foram calculadas as médias, desvios-padrão, medianas e valores mínimos e máximos dos dados sociodemográficos, como idade, escolaridade e renda em salários mínimos da época.

Realizaram-se análises bivariadas entre a variável dependente "risco de internação" - alto ou baixo, segundo o valor da PIR - e os grupos de variáveis independentes descritas anteriormente. As variáveis que mostraram associação com nível de significância $\leq 0,20$ foram avaliadas por meio de análise de regressão logística binária múltipla. Os blocos de variáveis independentes foram incorporados ao modelo, sequencialmente, pelo método stepforward, e o melhor ajuste do modelo foi avaliado mediante análise dos resíduos. As variáveis escolaridade, MEEM, EDG, índice de massa corporal, velocidade de marcha, força de preensão manual, AIVD e AVD entraram no modelo como numéricas. Os resultados de cada variável, antes e após ajustes por todas as demais, foram apresentados como razão de chance (OR), e seus intervalos de 95\% de confiança (IC95\%). Como idade e sexo são itens da variável dependente, não entraram no ajuste, evitando colinearidade. Utilizou-se o pacote estatístico SPSS versão 18.0 para Windows (SPSS Inc., Chicago, Estados Unidos).

Considerando-se o processo de amostragem inversa, pesos com ajustes foram utilizados como fatores de expansão para corrigir o fato de cada indivíduo selecionado representar a si mesmo e a outros que estão na população. Todas as análises apresentadas são ponderadas. Nas Tabelas 1, 2 e 3, os números absolutos em cada categoria de análise são apresentados sem ponderação.

\section{Resultados}

\section{Características da amostra estudada}

Dos 847 idosos que constituíam a amostra original do FIBRA-RJ, 81 (9,6\%) foram excluídos da análise pela impossibilidade de responderem a autoavaliação de saúde; dos 766 da amostra restante, dois idosos $(0,2 \%)$ foram considerados perda por não informarem o número de consultas no último ano, impossibilitando o cálculo da PIR. Foram analisados 764 indivíduos. A amostra caracterizou-se por ter maior proporção de mulheres, de cor de pele branca e de indivíduos casados; $54 \%$ apresentaram dependência para realizar pelo menos uma das AIVD, e $17 \%$ eram dependentes em pelo menos uma das AVD. As médias de idade, escolaridade e a mediana da renda em salários mínimos (SM) foram 76,8 anos $( \pm 6,8), 10,1$ anos $( \pm 5,1)$ e 4,9 SM $(0-53,1)$, respectivamente. Do total da amostra, $13,1 \%$ eram trabalhadores ativos, e $20,2 \%$ moravam sós. A amostra apresentou desempenho físico e cognitivo dentro da normalidade [mediana da velocidade da marcha $=5,3(2,0-68,3)$, média de força de preensão manual $=21,5( \pm 8,2) \mathrm{e}$ MEEM $=25,4( \pm 3,4)]$, porém a avaliação do humor $[E D G=7,0( \pm 1,7)]$ mostrou-se alterada. Tabelas 1 e 2 .

\section{Estratificação da amostra segundo o risco de internação}

Cinquenta idosos [6,7\% (IC95\%: 5,1-8,7)] apresentaram alto risco de internação. A média da PIR foi 0,31 (IC95\%: 0,30-0,32). A distribuição de frequência das oito variáveis que compõem o instrumento nos diferentes estratos de risco é apresentada na Tabela 3. Em relação ao grupo de baixo risco, o de alto risco apresentou pior avaliação de saúde - ruim/muito ruim; internou seis vezes mais; teve sete vezes mais coronariopatia e duas vezes mais diabetes mellitus; apresentou idade mais avançada e maior proporção de homens. Todos aqueles que não identificaram um cuidador em potencial, isto é, $12,7 \%$ da amostra, classificaram-se como de baixo risco.

As variáveis associadas ao risco de internação, na análise univariada e após ajuste do modelo, estão apresentadas na Tabela 4. Doença pulmonar obstrutiva crônica $(\mathrm{OR}=1,91)$, câncer $(\mathrm{OR}=2,30)$, ocorrência de quedas $(\mathrm{OR}=2,04) \mathrm{e}$ dependência para realizar AVD $(\mathrm{OR}=1,27)$ exerceram significativa influência no risco de internação, após ajustes para todas as outras condições; o mesmo se deu com o número de medicamentos utilizados $(\mathrm{OR}=1,45)$, necessidade de permanecer acamado no último ano $(\mathrm{OR}=5,06)$ 
Tabela 1

Características sociodemográficas e de independência nas atividades de vida diária da amostra, por estratos de risco ( $N=764)$. Rio de Janeiro, Brasil, 2009-2010.

\begin{tabular}{|c|c|c|c|}
\hline Dados sociodemográficos & $\begin{array}{c}\text { Total } \\
\text { n (\%) * }\end{array}$ & $\begin{array}{c}\text { Baixo risco } \\
\text { n (\%) * }\end{array}$ & $\begin{array}{c}\text { Alto risco } \\
\text { n (\%) * }\end{array}$ \\
\hline \multicolumn{4}{|l|}{ Sexo } \\
\hline Feminino & $537(67,1)$ & $515(95,8)$ & $22(4,2)$ \\
\hline Masculino & $227(32,9)$ & $199(88,2)$ & $28(11,8)$ \\
\hline Total & $764(100,0)$ & $714(93,3)$ & $50(6,7)$ \\
\hline \multicolumn{4}{|l|}{ Idade (anos) } \\
\hline $65-69$ & $137(17,1)$ & $135(98,7)$ & $2(1,3)$ \\
\hline $70-74$ & $185(22,9)$ & $179(96,4)$ & $6(3,6)$ \\
\hline $75-80$ & $187(25,5)$ & $171(91,1)$ & $16(8,9)$ \\
\hline $81-85$ & $152(21,2)$ & $145(95,3)$ & $7(4,7)$ \\
\hline$>85$ & $103(13,4)$ & $84(82,1)$ & $19(17,9)$ \\
\hline \multicolumn{4}{|l|}{ Raça/Cor de pele } \\
\hline Branca & $478(63,2)$ & $444(92,7)$ & $34(7,3)$ \\
\hline Mulata/Cabocla/Parda & $202(26,3)$ & $189(93,6)$ & $13(6,4)$ \\
\hline Outras & $84(10,6)$ & $81(96,3)$ & $3(3,7)$ \\
\hline \multicolumn{4}{|l|}{ Situação conjugal } \\
\hline Casado(a)/Com companheiro(a) & $329(44,3)$ & $308(93,3)$ & $21(6,7)$ \\
\hline Viúvo(a) & $291(37,5)$ & $273(94,0)$ & $18(6,0)$ \\
\hline Solteiro(a) & $85(10,7)$ & $79(92,3)$ & $6(8,9)$ \\
\hline Divorciado(a)/Separado(a) & $59(7,5)$ & $54(91,1)$ & $5(8,9)$ \\
\hline Morar só (sim) & $159(20,2)$ & $153(96,7)$ & $5(3,3)$ \\
\hline \multicolumn{4}{|l|}{ Escolaridade (anos) } \\
\hline Até 4 & $152(19,6)$ & $137(90,2)$ & $15(9,8)$ \\
\hline $5-8$ & $158(20,6)$ & $149(93,8)$ & $9(6,2)$ \\
\hline $9-12$ & $241(31,5)$ & $229(94,9)$ & $12(5,1)$ \\
\hline$>12$ & $213(28,2)$ & $199(93,3)$ & $14(6,7)$ \\
\hline \multicolumn{4}{|l|}{ Renda per capita (salários mínimos) ** } \\
\hline $0-2$ & $123(15,8)$ & $114(92,9)$ & $9(7,1)$ \\
\hline $2-5$ & $192(24,7)$ & $180(92,9)$ & $12(7,1)$ \\
\hline $5-10$ & $226(29,6)$ & $212(93,7)$ & $14(6,3)$ \\
\hline$>10$ & $130(17,6)$ & $122(94,2)$ & $8(5,8)$ \\
\hline \multicolumn{4}{|l|}{ Status funcional } \\
\hline Independência nas AIVD & $353(45,9)$ & $343(97,0)$ & $10(3,0)$ \\
\hline Independência nas AVD & $608(79,7)$ & $577(94,7)$ & $31(5,3)$ \\
\hline
\end{tabular}

AIVD: atividades instrumentais de vida diária; AVD: atividades básicas de vida diária.

* Os números absolutos são valores não ponderados; os percentuais são valores ponderados;

** Salário mínimo de referência: $\mathrm{R} \$ 465,00 ; 33$ idosos não informaram sua renda.

e necessidade de visita domiciliar de um profissional da saúde $(\mathrm{OR}=1,41)$. A condição de morar só $(\mathrm{OR}=0,59)$ e a independência para realizar as AIVD $(O R=0,89)$ associaram-se à redução do risco de internação. O MEEM $(\mathrm{OR}=1,05)$ e a força de preensão manual $(\mathrm{OR}=1,04)$ mostraram-se fracamente associados ao risco de internação, com os respectivos intervalos de confiança muito próximos da unidade, tendo ainda o sentido da associação conflitante após ajustes. A escolaridade, o IMC, a velocidade da marcha, presença de doenças articulares, AVC e distúrbios do sono não foram significativamente associados ao risco de internação. 
Tabela 2

Características funcionais da amostra por estratos de risco ( $N=764)$. Rio de Janeiro, Brasil, 2009-2010.

\begin{tabular}{lccc}
\hline Variáveis funcionais & Total da amostra & Baixo risco * & Alto risco ** \\
\hline MEEM [Média (DP)] & $25,4( \pm 3,4)$ & $25,5( \pm 3,4)$ & $24,8( \pm 3,4)$ \\
EDG [Média (DP)] & $7,0( \pm 1,7)$ & $7,0( \pm 1,7)$ & $7,7( \pm 2,0)$ \\
Velocidade de marcha (m/s) [Mediana (mínimo-máximo)] & $5,3(2,0-68,3)$ & $5,3(2,0-68,3)$ & $6,0(3,0-24,0)$ \\
Força de preensão manual (Kgf) [Média (DP)] & $21,5( \pm 8,2)$ & $21,5( \pm 8,1)$ & $21,1( \pm 8,1)$ \\
IMC (kg/m2) [Média (DP)] & $26,8( \pm 4,9)$ & $26,8( \pm 4,8)$ & $26,3( \pm 4,8)$ \\
\hline
\end{tabular}

DP: desvio-padrão; EDG: Escala de Depressão Geriátrica; IMC: índice de massa corporal; MEEM: Mini-Exame do Estado Mental.

* Probabilidade de internações repetidas (PIR) < 0,5;

$\star * \operatorname{PIR} \geq 0,5$

Tabela 3

Distribuição de frequência das variáveis que compõem o instrumento probabilidade de internações repetidas (PIR), por estratos de risco ( $N=764)$. Rio de Janeiro, Brasil, 2009-2010.

\begin{tabular}{|c|c|c|c|c|}
\hline Variáveis do PIR & $\begin{array}{c}\text { Baixo risco * } \\
\mathrm{n}(\%) \star \star \star\end{array}$ & $\begin{array}{l}\text { Alto risco ** } \\
n(\%) \star \star \star\end{array}$ & 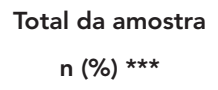 & Valor de $\mathrm{p}$ \\
\hline Autoavaliação de saúde & & & & 0,01 \\
\hline Muito boa & $109(15,3)$ & $3(6,8)$ & $112(14,8)$ & \\
\hline Boa & $294(41,5)$ & $21(43,1)$ & $315(46,1)$ & \\
\hline Regular & $281(39,1)$ & $17(32,7)$ & $298(38,6)$ & \\
\hline Ruim & $23(3,1)$ & $8(16,7)$ & $31(4,0)$ & \\
\hline Muito ruim & $7(1,0)$ & $1(0,7)$ & $8(1,0)$ & \\
\hline Idade (anos) & & & & 0,01 \\
\hline $75-79$ & $171(24,8)$ & $16(33,9)$ & $187(25,5)$ & \\
\hline $80-84$ & $145(21,7)$ & $07(14,8)$ & $152(21,2)$ & \\
\hline$>85$ & $84(11,8)$ & $19(35,7)$ & $103(13,4)$ & \\
\hline Internação \# & $94(13,0)$ & $42(83,6)$ & $136(17,7)$ & 0,01 \\
\hline$>6$ consultas \#\# & $262(36,3)$ & $38(76,7)$ & $300(39,0)$ & 0,01 \\
\hline Diabetes mellitus & $148(20,5)$ & $22(42,8)$ & $170(22,0)$ & 0,01 \\
\hline Coronariopatia & $68(9,7)$ & $35(71,1)$ & $103(13,8)$ & 0,01 \\
\hline Sem cuidador potencial & $99(13,6)$ & - & $99(12,7)$ & 0,01 \\
\hline Sexo masculino & $199(31,2)$ & $28(57,8)$ & $227(32,9)$ & 0,01 \\
\hline
\end{tabular}

* $\mathrm{PIR}<0,5$

** $\mathrm{PIR} \geq 0,5$;

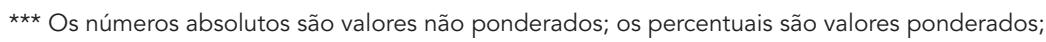

\# Pelo menos uma internação no último ano;

\#\# Consultas médicas no último ano.

\section{Discussão}

No presente estudo, $6,7 \%$ de uma amostra de idosos da zona norte da cidade do Rio de Janeiro apresentaram alto risco de internações repetidas. Esse risco foi associado à presença de do- enças crônicas como câncer e doença pulmonar obstrutiva crônica, ao uso de medicamentos, à presença de quedas, ao pior estado de saúde, caracterizado pela necessidade de permanecer acamado e de receber visita domiciliar de um profissional de saúde, e à dependência para 
Fatores associados ao risco de internação: modelo multivariado. Rio de Janeiro, Brasil, 2009-2010.

\begin{tabular}{|c|c|c|c|c|c|}
\hline Variáveis & OR bruto & IC95\% & $\begin{array}{c}\text { OR } \\
\text { ajustado }\end{array}$ & IC95\% & Valor de $\mathrm{p}$ \\
\hline \multicolumn{6}{|l|}{ Sociodemográficas } \\
\hline Escolaridade (anos) & 0,97 & $0,96-0,99$ & 0,98 & $0,96-1,00$ & 0,04 \\
\hline Morar só (sim) & 0,41 & $0,31-0,55$ & 0,59 & $0,43-0,88$ & 0,01 \\
\hline \multicolumn{6}{|l|}{ Funcionais } \\
\hline MEEM * & 0,96 & $0,95-0,98$ & 1,05 & $1,01-1,08$ & 0,01 \\
\hline EDG ** & 1,22 & $1,16-1,28$ & 1 & $0,64-1,05$ & 0,91 \\
\hline IMC & 0,98 & $0,96-0,99$ & 0,98 & $0,96-1,00$ & 0,01 \\
\hline Velocidade de marcha $(\mathrm{m} / \mathrm{s})$ & 1,05 & $1,03-1,10$ & 0,99 & $0,97-1,02$ & 0,93 \\
\hline Força de preensão manual (Kgf) & 0,99 & $0,98-1,06$ & 1,04 & $1,02-1,06$ & 0,01 \\
\hline AIVD *** & 0,83 & $0,78-0,82$ & 0,84 & $0,81-0,87$ & 0,01 \\
\hline AVD \# & 1,54 & $1,44-1,65$ & 1,27 & $1,07-1,51$ & 0,01 \\
\hline \multicolumn{6}{|l|}{ Saúde autorrelatada } \\
\hline Doença pulmonar obstrutiva crônica (sim) & 2,82 & $2,21-3,61$ & 1,91 & $1,44-2,55$ & 0,01 \\
\hline Artrose/Artrite (sim) & 1,57 & $1,32-1,86$ & 1,05 & $0,85-1,30$ & 0,05 \\
\hline Câncer (sim) & 3,37 & $2,57-4,43$ & 2,3 & $2,12-4,24$ & 0,01 \\
\hline $\operatorname{AVC}(\operatorname{sim})$ & 1,48 & $1,04-2,10$ & 0,79 & $0,53-1,17$ & 0,51 \\
\hline Quedas (sim) & 2,78 & $2,34-3,31$ & 2,04 & $1,66-2,50$ & 0,01 \\
\hline Distúrbios do sono (sim) & 1,46 & $1,23-1,73$ & 0,83 & $0,67-1,03$ & 0,52 \\
\hline \multicolumn{6}{|l|}{ Uso de serviços de saúde } \\
\hline Número de medicamentos & 1,27 & $1,23-1,30$ & 1,45 & $1,12-1,89$ & 0,01 \\
\hline Acamado no último ano (sim) & 7,42 & $6,20-8,88$ & 5,06 & $4,00-6,30$ & 0,01 \\
\hline Visita domiciliar no último ano (sim) & 4,16 & $3,42-5,07$ & 1,41 & $1,06-1,89$ & 0,01 \\
\hline
\end{tabular}

AIVD: atividades instrumentais de vida diária; AVC: acidente vascular cerebral; AVD: atividades básicas de vida diária;

DP: desvio-padrão; EDG: Escala de Depressão Geriátrica; IC95\%: intervalo de 95\% de confiança; IMC: índice de massa

corporal; MEEM: Mini-Exame do Estado Mental; OR: odds ratio.

* MEEM: 0 a 30;

** EDG: 0 a 15 ;

*** AIVD: 7 a 21

\# AVD: 0 a 6.

realizar as AVD. A condição de morar só e a independência nas AIVD associaram-se negativamente ao risco de internação.

Neste estudo, a proporção de idosos estratificados como de alto risco foi semelhante à de outros estudos nacionais e internacionais. Boult et al. ${ }^{5}$ encontraram proporção de $7,2 \%$ de idosos americanos de alto risco, não institucionalizados, beneficiários do programa Medicare, com idade maior ou igual a setenta anos. Wagner et al. 12, em estudo com idosos de 65 anos ou mais, não institucionalizados, realizado em três cidades europeias - Hamburgo (Alemanha), Londres (Reino Unido) e Solothurn (Suíça), encontraram $7 \%, 4 \%$ e $5 \%$ de idosos de alto risco, respectivamente. No Brasil, Guerra \& Ramos-Cerqueira 18 encontraram $6,2 \%$ de idosos de risco alto em uma amostra de 305 indivíduos com 65 anos ou mais, usuários de um Centro de Saúde Escola em Botucatu, São Paulo. Dutra et al. 19 encontraram prevalência de $7 \%$ em uma amostra populacional da cidade de Progresso, Rio Grande do Sul. Por outro lado, Veras et al. 20 encontraram 2,5\% de idosos classificados como de alto risco entre 606 indivíduos com idade a partir de 65 anos, que procuraram o guichê de recepção de um ambulatório da rede pública da cidade do Rio de Janeiro, no ano 2000. Ainda, Estrella et al. 21 avaliaram 1.423 idosos pertencentes à carteira de uma operadora de saúde na cidade do Rio de Janeiro, os quais responderam o questionário aplicado via telefone. Os autores encontraram frequência de $3,2 \%$ de idosos de alto risco. As características da população-fonte dos estudos citados podem 
justificar a diferença das proporções encontradas. Observa-se que, nos estudos de base populacional, a fração de idosos de alto risco é maior quando comparada aos demais, provavelmente por permitir que idosos com pouca mobilidade e, portanto, em piores condições de saúde fossem abordados.

Algumas associações encontradas no presente estudo são bem estabelecidas e descritas na literatura, como no caso da relação entre a presença de morbidades e quedas com a síndrome de fragilidade 22,23. Rockwood \& Mitnitski 24 conceituam fragilidade como acúmulo de déficits ao longo da vida e computam, para formular o seu índice, as doenças crônicas, entre outros parâmetros. $\mathrm{O}$ escore do índice de fragilidade de Rockwood \& Mitnitski 24 relaciona-se positivamente com mortalidade, institucionalização e internações prolongadas. O uso de medicamentos, amplamente relacionado à hospitalização em idosos 25 , também se associou ao risco de internação. Apesar de a capacidade funcional ter apresentado influência no risco de internação, as medidas de desempenho não mostraram associações significativas ou robustas, tanto no domínio mental (cognição e humor), como no físico (velocidade de marcha e força de preensão manual), itens que integram alguns instrumentos para identificação da fragilidade 26. Essa observação desperta uma reflexão sobre o que se encontra na literatura a respeito da importância da diversidade de instrumentos de rastreio de idosos frágeis, em face da complexidade dessa síndrome, com suas diferentes facetas. O objetivo da realização do rastreamento, seja clínico, seja epidemiológico, seja para planejamento e gestão dos recursos da saúde, é que deve determinar a escolha do instrumento.

No presente estudo, o arranjo unipessoal de moradia associou-se a um menor risco de internação. Esse é um achado interessante, visto que, no Brasil, como em outros países, verifica-se aumento no número de idosos que residem sozinhos. Segundo Camargos et al. 27, as melhorias na condição de renda, na capacidade funcional e na saúde em geral parecem ser determinantes na decisão de viver sozinho entre idosos. É possível, portanto, que a autossuficiência, considerada na fórmula da PIR como uma interpretação às tendências de resposta ao item suporte social, talvez seja mais bem representada, no Brasil, pela variável "morar só".

O instrumento PIR foi proposto originalmente para rastrear idosos que pudessem se beneficiar de intervenções para redução dos desfechos da fragilidade 5. No Brasil, os estudos aqui pontuados descreveram perfis de risco, em amostras de conveniência e em diversos ambientes de serviços de saúde 18,19,20,21. Em todos eles, o instrumento mostrou-se operacionalmente adequado para estratificar idosos, isto é, sua simplicidade tornou fácil o treinamento de examinadores e foi capaz de reproduzir resultados que se assemelharam, em suas proporções, àqueles encontrados em outras realidades. Apesar das evidências de sua utilidade para uso em nosso meio, deve-se considerar que o instrumento foi criado a partir de variáveis preditivas baseadas em dados empíricos de amostras da população norte-americana. Por essa razão - e também porque populações com diferenças fenotípicas marcantes produzem diferentes apresentações nosológicas -, deve-se considerar a possibilidade de, por meio de estudos longitudinais com amostras populacionais nacionais, identificarmos variáveis potencialmente mais adequadas no rastreio dos idosos brasileiros. Da mesma maneira, os pesos relativos associados a cada variável, no instrumento proposto por Boult et al. 5, podem ter ponderações distintas em realidades diversas, tais como a brasileira.

Por exemplo, no presente estudo, quando avaliados os itens que compõem o instrumento PIR, observou-se que $12,7 \%$ do total da amostra relataram ausência de suporte social, e todos esses indivíduos foram classificados como de baixo risco. De modo semelhante, no estudo realizado em Botucatu, apenas nove dos 305 idosos estudados não apresentaram apoio social, e todos foram classificados como de baixo risco ${ }^{18}$. Ao ser inserido na fórmula do PIR, esse item (ausência da identificação de um cuidador) confere redução no risco de internação, sendo interpretado como um sinal de autossuficiência do indivíduo estudado, segundo os autores do instrumento 3. Entretanto, sabe-se da importância do suporte social como fator protetor de eventos desfavoráveis para os idosos brasileiros 28 e do papel da família como fonte informal direta de apoio 29. No Rio de Janeiro, segundo o estudo de Veras et al. 20, 93,7\% dos idosos identificaram cuidadores. Portanto, observa-se que a frequência e a distribuição desse item sugerem possíveis diferenças de significado quando o instrumento é aplicado em um contexto sociocultural distinto do norte-americano. A identificação de uma variável ("morar só") que talvez possa substituir a original ("a possibilidade de identificar algum amigo, parente ou vizinho como cuidador"), que parece não apresentar o mesmo significado no contexto sociocultural brasileiro, foi considerada um aspecto positivo deste estudo.

A autoavaliação de saúde, um dos itens do instrumento PIR, é uma medida subjetiva e multidimensional que o indivíduo tem da qualidade de sua saúde física e mental, sendo um dos indi- 
cadores mais utilizados em pesquisas gerontológicas por predizer desfechos desfavoráveis, como mortalidade e dependência funcional. A adequação do uso de informações sobre a percepção de saúde obtidas por intermédio de um respondente substituto ainda é objeto de estudo, e pesquisadores divergem no modo como as utilizam, por vezes, excluindo-as, em outras, mantendo-as 30. No presente estudo, os indivíduos com necessidade de informante substituto foram excluídos, seguindo o mesmo critério utilizado por Boult et al. 5 em seu trabalho original. Estes idosos constituem um subgrupo da população que apresenta evidentes sinais não só de fragilidade, como também dos seus desfechos, devendo ser objeto de análises específicas futuramente.

Entre as limitações do presente trabalho, deve-se considerar que a frequência de alguns dados, como as doenças crônicas, as quedas, o uso de serviços de saúde, entre outros, pode ter sido subestimada, uma vez que o autorrelato talvez tenha sofrido o efeito da dificuldade de recordação, influenciando as análises de associação. Além disso, o desenho transversal não permite estabelecer relações causais entre as diversas variáveis estudadas e o risco de internações repetidas.

Outro aspecto que merece destaque é a modificação das opções de resposta da autoavaliação de saúde, tendo sido adotado o padrão das pesquisas censitárias brasileiras. Para que o cálculo sofresse menor impacto, optou-se pelas seguintes medidas: utilizaram-se todos os coeficientes de regressão e a simetria originais, atribuindo-se pesos iguais às opções "ruim" e "muito ruim", esta última inexistente originalmente. Considerando-se a baixa frequência da reposta "muito ruim" e a semelhança entre os resultados da estratificação do risco e os de outros estudos, o instrumento parece ter mantido as suas características. Entretanto, é necessário que o estudo de validação com a determinação das propriedades psicométricas do instrumento seja realizado. Até que dados relativos ao acompanhamento dessa população sejam gerados, deve-se refletir com cuidado sobre os resultados obtidos. Além disso, o fato de ter avaliado uma amostra de indivíduos beneficiários de uma operadora de saúde, portanto com menor restrição de acesso a serviços de saúde, não permite generalizações para o conjunto da população brasileira, que, em sua maioria, ainda sofre as restrições de acesso a esses serviços, impostas pela inadequação de oferta no setor público. Por outro lado, no Brasil, e especialmente no Sudeste, há um crescente número de usuários do sistema de saúde suplementar, que constituem uma população muito pouco estudada quanto às suas características básicas de risco 13 .

Como perspectivas futuras, a coorte que se inaugura - e da qual trata o presente artigo - permitirá o acompanhamento mais detalhado das variáveis estudadas, possibilitando o estudo da acurácia do instrumento PIR e, ainda, propor e testar algumas adaptações deste para uso no Brasil.

\section{Resumen}

El objetivo del estudio fue determinar el perfil de riesgo y los factores asociados con la fragilidad en la tercera edad. El origen de la población, compuesta por personas de 65 años o más, son los diferentes barrios de la ciudad de Río de Janeiro, Brasil, y clientes de planes de salud. El estudio fue transversal en una cohorte con una muestra estratificada por sexo y edad, compuesta de 764 individuos. Para la estratificación de riesgo se utilizó como instrumento la probabilidad de hospitalizaciones repetidas. Se realizó un análisis de regresión logística para estudiar la asociación entre el riesgo y un conjunto de variables sociodemográficas, estado de salud, funcionales y cognitivas. Se halló un 6,7\% de los pacientes ancianos con alto riesgo de hospitalización. El cáncer, caídas, enfermedad pulmonar obstructiva crónica, los medicamentos utilizados, recibir visitas de profesionales de la salud, estar postrado en cama en su casa, vivir solo y actividades de la vida diaria se asociaron con el riesgo de hospitalización. El instrumento parece ser útil para estratificar el riesgo en ancianos.

Hospitalización; Envejecimineto; Salud del Anciano; Servicios de Salud 


\section{Colaboradores}

M. Perez participou da concepção do estudo, aquisição, análise e interpretação dos dados e redação do artigo. R. A. Lourenço colaborou na concepção e planejamento do estudo, revisão crítica e aprovação da versão final antes do envio para publicação.

\section{Agradecimentos}

À Maria Angélica Sanchez, pela coordenação do trabalho de campo, e à equipe de auxiliares de pesquisa do Laboratório de Pesquisa em Envelhecimento Humano da Universidade do Estado do Rio de Janeiro. Ao CNPq (processo 555087/2006-9) e à FAPERJ (processo E26/171.489/2006), pelo financiamento.

\section{Referências}

1. Silva ZP, Ribeiro MCSA, Barata RB, Almeida MF. Perfil sociodemográfico e padrão de utilização dos serviços de saúde do Sistema Único de Saúde (SUS), 2003-2008. Ciênc Saúde Coletiva 2011; 16:3807-16.

2. Veras RP. Envelhecimento populacional contemporâneo: demandas, desafios e inovações. Rev Saúde Pública 2009; 43:548-54.

3. Fried LP, Tangen CM, Walston J, Newman AB, Hirsch C, Gottdiener J, et al. Frailty in older adults: evidence for a phenotype. J Gerontol A Biol Sci Med Sci 2001; 56:M146-56

4. Hogan DB, MacKnight C, Bergman H; Steering Committee, Canadian Initiative on Frailty and Aging. Models, definitions and criteria of frailty. Aging Clin Exp Res 2003; 15(3 Suppl):1-29.

5. Boult C, Dowd B, McCaffrey D, Boult L, Hernandez R, Krulewitch H. Screening elders for risk of hospital admission. J Am Geriatr Soc 1993; 41:811-7.

6. Boult L, Boult C, Pirie P, Pacala JT. Test-retest reliability of a questionnaire that identifies elders at risk for hospital admission. J Am Geriatr Soc 1994 42:707-11.

7. Boult C, Boult L, Morishita L, Smith SL, Kane RL. Outpatient geriatric evaluation and management. J Am Geriatr Soc 1998; 46:296-302.

8. Boult C, Boult L, Morishita L, Dowd B, Kane RL, Urdangarin CF. A randomized clinical trial of outpatient geriatric evaluation and management. J Am Geriatr Soc 2001; 49:351-9.
9. Pacala JT, Boult C, Boult L. Predictive validity of a questionnaire that identifies older persons at risk for hospital admission. J Am Geriatr Soc 1995; 43:374-77.

10. Sidorov J, Shull R. "My patients are sicker": using the PRA risk survey for case finding and examining primary care site utilization patterns in a medicare-risk MCO. Am J Manag Care 2002; 8:569-75.

11. Vojta CL, Vojta DD, TenHave TR, Amaya M, Lavizzo-Mourey R, Asch DA. Risk screening in a Medicare/Medicaid Population. J Gen Intern Med 2001; 16:525-30.

12. Wagner JT, Bachmann LM, Boult C, Harari DW, Egger M, Beck JC, et al. Predicting the risk of hospital admission in older persons - validation of a brief self-administered questionnaire. J Am Geriatr Soc 2006; 54:1271-6.

13. Veras RP, Caldas CP, Araújo DV, Mendes RKW. A assistência suplementar de saúde e seus projetos de cuidado para com o idoso. Ciênc Saúde Coletiva 2008; 13:1119-26.

14. Brucki SMD. Sugestão para uso do Mini-Exame do Estado Mental no Brasil. Arq Neuropsiquiatr 2003; 61:777-81.

15. Paradela EMP, Lourenço RA. Validação da escala de depressão geriátrica em ambulatório geral. Rev Saúde Pública 2005; 39:918-23.

16. Lawton M, Brody P. Assessment of older people: self-maintaining and instrumental activities of daily living. Gerontologist 1969; 9:179-86. 
17. Katz S, Ford AB, Moskowitz RW, Jackson BA, Jaffe MW. Studies of illness in the aged. The index of ADL: a standardized measure of biological and psychosocial function. JAMA 1963; 185:914-9.

18. Guerra IC, Ramos-Cerqueira ATA. Risco de hospitalizações repetidas em idosos usuários de um centro de saúde escola. Cad Saúde Pública 2007; 23:585-92.

19. Dutra MM, Moriguchi EH, Lampert MA, Poli-deFigueiredo CE. Validade preditiva de instrumento para identificação do idoso em risco de hospitalização. Rev Saúde Pública 2011; 45:106-12.

20. Veras RP, Lourenço RA, Martins CSF, Sanchez MAS, Chaves PHC. Novos paradigmas do modelo assistencial no setor saúde: consequências da explosão populacional dos idosos no Brasil. In: Veras RP, organizador. Gestão contemporânea em saúde: terceira idade. Rio de Janeiro: Editora RelumeDumará/Universidade Aberta da Terceira Idade, Universidade do Estado do Rio de Janeiro; 2002. p. 11-79.

21. Estrella K, Oliveira CEF, Sant'Anna AA, Caldas CP. Detecção do risco para internação hospitalar em população idosa: um estudo a partir da porta de entrada no sistema de saúde suplementar. Cad Saúde Pública 2009; 25:507-12.

22. Chang SS, Weiss CO. Association between inflammatory-related disease burden and frailty: results from the Women's Health and Aging Studies (WHAS) I and II. Arch Gerontol Geriatr 2012; 54: 9-15.

23. Kinney JM. Nutritional frailty, sarcopenia and falls in the elderly. Curr Opin Clin Nutr Metab Care 2004; 7:15-20.
24. Rockwood K, Mitnitski A. Frailty in relation to the accumulation of deficits. J Gerontol A Biol Sci Med Sci 2007; 62:722-7.

25. Budnitz DS, Lovegrove MC, Shehab N, Pharm D. Emergency hospitalizations for adverse drug events in older Americans. N Engl J Med 2011; 365:2002-12.

26. Sternberg SA, Wershof SA, Karunananthan S, Bergman H, Clarfield M. A identification of frailty: a systematic literature review. J Am Geriatr Soc 2011; 59:2129-38.

27. Camargos MCS, Rodrigues RN, Machado CJ. Idoso, família e domicílio: uma revisão narrativa sobre a decisão de morar sozinho. Rev Bras Estud Popul $2011 ; 28: 217-30$

28. d'Orsi E, Xavier AJ, Ramos LR. Trabalho, suporte social e lazer protegem idosos da perda funcional: Estudo Epidoso. Rev Saúde Pública 2011; 45:685-92.

29. Camarano AA, Kanso S, Mello JL, Pasitano MT. Famílias: espaço de compartilhamento de recursos e vulnerabilidades. In: Camarano AA, organizadora. Os novos idosos brasileiros. Rio de Janeiro: Instituto de Pesquisa Econômica Aplicada; 2004. p. 137-67.

30. Lima-Costa MF, Peixoto SV, Matos DL, Firmo JOA, Uchôa E. A influência de respondente substituto na percepção da saúde de idosos: um estudo baseado na Pesquisa Nacional por Amostra de Domicílios $(1998,2003)$ e na coorte de Bambuí, Minas Ge rais, Brasil. Cad Saúde Pública 2007; 23:1893-902.

Recebido em 31/Mai/2012

Versão final reapresentada em 13/Fev/2013

Aprovado em 07/Mar/2013 